\title{
miR-574-5p attenuates proliferation, migration and EMT in triple-negative breast cancer cells by targeting BCL11A and SOX2 to inhibit the SKIL/TAZ/CTGF axis
}

\author{
KE-JING ZHANG ${ }^{1,2}$, YU HU ${ }^{1,2}$, NA LUO ${ }^{1,2}$, XIN LI $^{1,2}$, FEI-YU CHEN ${ }^{1,2}$, JIA-QI YUAN ${ }^{1,2}$ and LEI GUO ${ }^{1,2}$ \\ ${ }^{1}$ Department of Breast Surgery, Xiangya Hospital, Central South University; ${ }^{2}$ Clinical Research Center For \\ Breast Cancer Control and Prevention In Hunan Province, Xiangya Hospital, Changsha, Hunan 410008, P.R. China
}

Received July 16, 2019; Accepted November 21, 2019

DOI: $10.3892 /$ ijo.2020.4995

\begin{abstract}
Triple-negative breast cancer (TNBC) is a subtype of breast cancer with a high degree of malignancy. TNBC is prone to distant metastasis and has a poor prognosis. A number of TNBC-related microRNAs (miRNAs) have been studied and identified. However, the detailed roles of miR-574-5p in TNBC remain poorly understood. miR-574-5p, SRY (sex determining region Y)-box 2 (SOX2), B-cell lymphoma/leukaemia 11A (BCL11A), SKI like proto-oncogene (SKIL) and epithelial-mesenchymal transition (EMT)-related miRNAs and proteins were measured by reverse transcription-quantitative PCR and western blotting analysis, respectively. A luciferase reporter assay was employed to validate the direct targeting of SOX2 and BCL11A by miR-574-5p. MTT, colony formation and Transwell assays were performed to analyse the biological functions of miR-574-5p in TNBC cells. A nude mouse xenograft model was used to verify the effects of miR-574-5p on the tumorigenesis of TNBC in vivo. The results demonstrated that miR-574-5p levels were decreased in breast cancer tissues and cells. miR-574-5p repressed proliferation, migration and EMT in TNBC cells. Further experiments confirmed that miR-574-5p reduced tumour size and metastasis in vivo. miR-574-5p targeted BCL11A and SOX2 to inhibit the SKIL/transcriptional co-activator with PDZ-binding motif/connective tissue growth factor axis, and the inhibitory effect of miR-574-5p in TNBC cells was at least partly dependent on SOX2 and BCL11A. In addition, the regulation of downstream oncogenes by SOX2 was dependent on BCL11A. To the best of our knowledge,
\end{abstract}

Correspondence to: Dr Lei Guo, Department of Breast Surgery, Xiangya Hospital, Central South University, 87 Xiangya Road, Changsha, Hunan 410008, P.R. China

E-mail: guolei1346@163.com

Key words: triple-negative breast cancer, microRNA-574-5p, SRY (sex determining region Y)-box 2, B-cell lymphoma/leukaemia 11A, SKI like proto-oncogene, epithelial-mesenchymal transition this is the first study to report the association between the miR-574-5p/BCL11A/SOX2 axis and the tumorigenesis of TNBC, which provides a new mechanism for understanding the progression of TNBC.

\section{Introduction}

Of female malignant tumour types, breast cancer is one of the most common and has the highest incidence, which seriously endangers women's health $(1,2)$. Triple-negative breast cancer (TNBC) refers to a subtype of breast cancer that is negative for oestrogen receptor, progesterone receptor and human epidermal growth factor receptor 2 expression, accounting for $10-20 \%$ of all breast cancer cases (3). Compared with other types of breast cancer, TNBC has a poor prognosis (4). Of the pathological types, most are invasive ductal carcinoma, which is characterized by a strong invasiveness, high recurrence rate, high metastasis rate, short survival time and high mortality (5). Investigating the pathogenesis of TNBC can provide a theoretical basis for improving its treatment. However, the molecular regulatory mechanisms of TNBC development remain unclear.

B-cell lymphoma/leukaemia 11A (BCL11A) was first found in B cell chronic lymphocytic leukaemia. Its coding region encodes a transcription factor activating protein with a Kruppel zinc finger structure $(6,7)$. A previous study by our group has shown that BCL11A promotes TNBC stemness and tumorigenesis (8). However, as a transcription factor cofactor, the molecular mechanism by which it interacts with transcription factors to promote TNBC metastasis remains to be studied. A recent study has reported that in lung cancer, BCL11A is involved in the transcriptional upregulation of pro-cancer factors by directly binding to SRY (sex determining region Y)-box 2 (SOX2) (9). SOX2 is a factor associated with a variety of processes, such as cancer cell stemness and epithelial-mesenchymal transition (EMT) (10-12). However, whether this regulation is also present in TNBC remains to be studied.

The SKI like proto-oncogene (SKIL; also termed SnoN or SnoA) is a gene that was discovered in the 1980s, which belongs to the SKI family of intranuclear oncoproteins (13). Studies have shown that SKIL gene expression is increased in numerous tumour types, such as oesophageal cancer, ovarian cancer, melanoma, lung cancer and breast cancer (14-19). 
SKIL serves a dual role in the development of breast cancer. Zhu et al (20) found that inhibition of SKIL expression promoted the metastasis of breast cancer to the bone and lung, while Band and Laiho (21) showed that high expression of SKIL promoted the invasion and metastasis of breast cancer. A recent study has demonstrated that SKIL is regulated by transcription factor SOX2, and SKIL can promote breast cancer proliferation, migration and EMT by regulating the Hippo pathway (22). Therefore, the mechanism of SKIL in breast cancer deserves further study.

Transcriptional co-activator with PDZ-binding motif (TAZ), a classical effector protein of the Hippo pathway, mediates different tissue growth. As an oncogene, unrestrained TAZ activity is reported to counteract classical tumor suppressor checkpoints (23). TAZ is usually highly expressed in many cancer types, including breast cancer cells, and as the final effector of the Hippo pathway, TAZ is an attractive target in tumor treatment (24). Connective tissue growth factor (CTGF), a well-known oncogene involved in EMT and tumour metastasis, is a direct target gene of TAZ (22). Zhu et al (22) found that SKIL could enhance the transcription and oncogenic activity of TAZ and that SKIL-knockdown could lead to the downregulation of TAZ and its target CTGF.

MicroRNAs (miRNAs) are a class of endogenous non-coding small RNAs that were discovered in 1993; they are 22 nucleotides in length and are encoded by endogenous genes $(25,26)$. miRNAs can be involved in numerous biological pathways, such as embryonic development, cell proliferation, cell differentiation, growth and apoptosis (27-29). miR-574 is a tumour suppressor miRNA, and it has been reported that the long non-coding RNA linc-ZNF469-3 can promote breast cancer by inhibiting miR-574 (30). However, there are still few reports on the mechanism of miR-574 in breast cancer, and its regulation of EMT remains unclear. Bioinformatics analysis performed in the present study revealed that miR-574 can simultaneously target BCL11A and SOX2. Therefore, the present study hypothesized that miR-574-5p may inhibit the expression of SKIL by targeting BCL11A and SOX2, thereby inhibiting the proliferation, migration and EMT of TNBC.

In summary, the present study investigated the role of miR-574-5p in TNBC by studying the regulatory relationship and mechanism of miR-574-5p on the BCL11A/SOX2 axis. The current study provides novel ideas to further the understanding of TNBC.

\section{Materials and methods}

Clinical samples and cell lines. A total of 45 pairs of $\mathrm{BC}$ and respective adjacent normal tissues were obtained from 45 female patients (mean age, 55.4 years; age range, 24-76 years) who underwent surgery at the Department of Breast Surgery, Xiangya Hospital, Central South University (Changsha, China) between January 2017 and February 2018. Adjacent tissue samples were obtained within $3 \mathrm{~cm}$ of the edge of the cancer tissue. The 45 paired BC samples were divided into TNBC $(n=16)$ and non-TNBC $(n=29)$ based on immunohistochemistry staining of estrogen receptor (ER), progesterone receptor (PR) and human epidermal growth factor receptor (HER-2), and according to the Chinese Society of Clinical Oncology Breast Cancer Diagnosis and Treatment Guidelines 2018 (31).
No preoperative radiotherapy, chemotherapy or other tumour treatments were performed prior to tumour resection. Informed consent was signed by all patients, and this research was approved by the Ethics Committee of Xiangya Hospital Affiliated to Central South University (approval no. 201803377). MCF10A, MCF7, T47D, MDA-MB-436, MDA-MB-231, MDA-MB-468, SK-BR-3 cell lines were purchased from American Type Culture Collection and identified by STR verification. The MDA-MB-436, MDA-MB-231 and MDA-MB-468 cells were TNBC cell lines. The cells were cultured in DMEM (Invitrogen; Thermo Fisher Scientific, Inc.) supplemented with $10 \%$ foetal bovine serum (FBS; Invitrogen; Thermo Fisher Scientific, Inc.), 1\% streptomycin (Invitrogen; Thermo Fisher Scientific, Inc.) and $1 \%$ penicillin (Invitrogen; Thermo Fisher Scientific, Inc.) in a $5 \% \mathrm{CO}_{2}$ atmosphere at $37^{\circ} \mathrm{C}$.

Immunohistochemistry. Tumor tissue samples were fixed with $10 \%$ formalin for $24 \mathrm{~h}$ at $4^{\circ} \mathrm{C}$, embedded in paraffin and sectioned to a thickness on $2 \mu \mathrm{m}$. The sections were then deparaffinized and blocked with $10 \%$ goat serum (Gibco; Thermo Fisher Scientific, Inc.) in PBS for $1 \mathrm{~h}$ at $4^{\circ} \mathrm{C}$. The slides were incubated with anti-ER (1:100; Abcam; cat. no. ab108398), anti-PR (1:100; Abcam; cat. no. ab32085) and anti-HER-2 (1:100; Abcam; cat. no. ab32085) for $12 \mathrm{~h}$ at $4^{\circ} \mathrm{C}$. Following washing with PBS, the sections were incubated with a goat anti-rabbit HRP-conjugated secondary antibody (1:10,000; cat. no. 4414; Cell Signalling Technology, Inc.) for an additional $30 \mathrm{~min}$ at $25^{\circ} \mathrm{C}$. Following staining with hematoxylin for $5 \mathrm{~min}$ at $25^{\circ} \mathrm{C}$, the slices were blocked with neutral gum at $25^{\circ} \mathrm{C}$ for $24 \mathrm{~h}$ and images were obtained using a light microscope (magnification, x100). Non-specific primary antibody staining was assessed by substituting the primary antibody with PBS, and at least 10 fields were randomly selected from each section for evaluation. For scoring, ER negative and PR negative were defined as $<1 \%$ of cells with positive ER and positive PR staining based on five independent fields. HER-2- or HER-2 ${ }^{+}$ were considered to be negative for HER-2. HER-2- was when the pathologist observed no staining or membrane staining in $<10 \%$ of the tumour cells. HER-2 $2^{+}$was when faint or barely perceptible membrane staining was detected in $>10 \%$ of tumour cells. A sample was considered HER-2 ${ }^{++}$when the pathologist observed a weak-to-moderate complete membrane staining in $>10 \%$ of the tumour cells. HER-2 $2^{++}$samples were applied for fluorescence in situ hybridization, and the cases with amplification were defined as HER2-positive. The samples negative for all three markers were defined as TNBC, whereas all other cases were considered non-TNBC.

Fluorescence in situ hybridization. HER-2 ${ }^{++}$samples were applied for fluorescence in situ hybridization. The ZytoLight ${ }^{\circledR}$ SPEC ERBB2/CEN 17 Dual Color Probe (Zytomed, Systems $\mathrm{GmbH})$, which employs two DNA probes, was used to perform the assay, according to the manufacturer's protocol. The following probes were used: HER-2(ERBB2) gene specific probe, ZyGreen (excitation $503 \mathrm{~nm} / \mathrm{emission} 528 \mathrm{~nm}$ )-labelled polynucleotides $(\sim 10.0 \mathrm{ng} / \mu \mathrm{l})$, which target sequences mapping in 17q12-q21.1* (chr17:37,572,531-38,181,308) harbouring the ERBB2 gene region; and an $\alpha$ satellite probe targeting the centromere region of chromosome 17 (CEP17): ZyOrange 
(excitation $547 \mathrm{~nm} / \mathrm{emission} 572 \mathrm{~nm}$ )-labelled polynucleotides $(\sim 1.5 \mathrm{ng} / \mu \mathrm{l})$, which target sequences mapping in 17p11.1-q11.1 specific for the alpha satellite centromeric region D17Z1 of chromosome 17 . In brief, specimens were incubated with pre-treatment solution $\left(99^{\circ} \mathrm{C}\right.$ for $\left.20 \mathrm{~min}\right)$ and then digested with protease $\left(37^{\circ} \mathrm{C}\right.$ for $\left.10 \mathrm{~min}\right)$. The DNA probe was applied and hybridized to each section overnight at $37^{\circ} \mathrm{C}$. The slides were then washed, counterstained with DAPI, and observed by fluorescence microscopy. In each of the specimens of papillary carcinoma, the total numbers of HER2 and CEP17 signals were counted in 20 tumour cell nuclei, and the ratios of HER2 signals to CEP17 signals were calculated. Polysomy 17 was defined as the occurrence of a centromere copy number of three or more for chromosome 17 per cell.

Prediction targeting sequence. Possible miR-574-5p targets were predicted by bioinformatics analysis with TargetScan (version 7.2; http://www.targetscan.org/vert_72/).

Plasmid construction and transfection. Short hairpin RNA (shRNA)-negative control (NC), shRNA-BCL11A (shBCL11A), NC mimic and miR-574-5p mimic (for miR-574-5p overexpression) were purchased from Shanghai GenePharma Co., Ltd. The target sequences of the shRNA are presented in Table I. The miRNA mimics $(2 \mu \mathrm{g}, 100 \mathrm{nM})$ or shRNA $(1 \mu \mathrm{g}, 100 \mathrm{nM})$ were transfected into MDA-MB-468 or MDA-MB-231 cells using Lipofectamine ${ }^{\circledR} 3000$ (Invitrogen; Thermo Fisher Scientific, Inc.), according to the manufacturer's protocol. For SOX2 and BCL11A overexpression, the pcDNA3.1 vector (Shanghai GenePharma Co., Ltd.) was used to construct the recombinant plasmids pcDNA3.1-SOX2 and pcDNA3.1-BCL11A, respectively. The recombinant vectors (1 $\mu \mathrm{g}, 100 \mathrm{nM}$ ) were transfected into MDA-MB-468 or MDA-MB-231 cells using Lipofectamine ${ }^{\circledR} 3000$, according to the manufacturer's protocol. A total of $48 \mathrm{~h}$ after transfection, cells were used for subsequent experiments.

MTT assay. The MTT experiment was applied to analyse cell viability. MDA-MB-468 and MDA-MB-231 cells were seeded in 96-well plates $\left(5 \times 10^{4}\right.$ cells $\left./ \mu 1\right)$. Subsequently, $0.5 \%$ MTT (Sigma-Aldrich; Merck KGaA) was added to each well and incubated for $4 \mathrm{~h}$. Then, DMSO $(150 \mu \mathrm{l})$ was added to each well and incubated for $10 \mathrm{~min}$. The absorbance at $490 \mathrm{~nm}$ was detected using the Thermo Fisher Multiskan FC plate reader (Thermo Fischer Scientific, Inc.).

Colony formation assay. MDA-MB-468 and MDA-MB-231 cells in the logarithmic growth phase were serially diluted and then inoculated separately in culture dishes and cultured at $37^{\circ} \mathrm{C}$ for 2 weeks. The cells were fixed with a mixture of acetic acid and formic acid (at a ratio of 1:3) for $15 \mathrm{~min}$ at $25^{\circ} \mathrm{C}$, stained with Giemsa solution for $15 \mathrm{~min}$ at $25^{\circ} \mathrm{C}$ and then rinsed slowly with running water. Colonies were counted using a light microscope (magnification, x40). Five fields were randomly selected and counted for each well.

Transwell assay. Cells were seeded into the upper chamber (1.0x10 ${ }^{6}$ cells/chamber). RPMI-1640 medium (Gibco; Thermo Fisher Scientific, Inc.) with $10 \%$ FBS was added to the lower chamber. Cells were incubated in the chambers at $37^{\circ} \mathrm{C}$ for
$48 \mathrm{~h}$. The lower chamber was stained with $0.1 \%$ crystal violet $30 \mathrm{~min}$ at $25^{\circ} \mathrm{C}$, and the cells were counted under a light microscope (magnification, x100). Five fields were randomly selected and counted for each well. For the cell migration assay, 24-well plates (8- $\mu \mathrm{m}$ pores; Corning, Inc.) without coating were used. For the invasion assay, the upper chambers were coated with Matrigel (1:8 dilution; BD Biosciences).

Total RNA extraction and reverse transcription-quantitative $P C R(R T-q P C R)$. Total RNA was extracted using TRIzol (Thermo Fisher Scientific, Inc.). The PrimeScript RT Reagent kit with gDNA Eraser (Takara Bio, Inc.) and the SYBR Green qPCR Master mix kit (Takara Bio, Inc.) were used for RT $\left(45 \mathrm{~min}\right.$ at $50^{\circ} \mathrm{C}$ ) and $\mathrm{qPCR}$, respectively. The thermocycling conditions were as follows: $96^{\circ} \mathrm{C}$ for $6 \mathrm{~min}$, followed by 40 cycles of $96^{\circ} \mathrm{C}$ for $15 \mathrm{sec}, 57^{\circ} \mathrm{C}$ for $30 \mathrm{sec}$ and $72^{\circ} \mathrm{C}$ for $30 \mathrm{sec}$. GADPH was used as an internal control for SOX2 and BCL11A. U6 was used as an internal control for miRNA-574-5p. The $2^{-\Delta \Delta \mathrm{Cq}}$ method (32) was used to calculate the relative expression level. The primer sequences are listed in Table II.

Western blot analysis. Total protein of MDA-MB-468 or MDA-MB-231 cells was extracted using RIPA buffer (Invitrogen; Thermo Fisher Scientific, Inc.). The protein concentration was measured using a BCA Protein assay kit (Takara Bio, Inc.). Protein $(30 \mu \mathrm{g})$ was separated by $10 \%$ SDS-PAGE at $40 \mathrm{~V}$ for $4-5 \mathrm{~h}$ and then transferred to a PVDF membrane (Sigma-Aldrich; Merck KGaA). The PVDF membrane was stained with 1x Ponceau S solution (Beijing Solarbio Science \& Technology, Co., Ltd.) for $5 \mathrm{~min}$. The membranes were then blocked with $5 \%$ non-fat milk at room temperature for $1 \mathrm{~h}$ and incubated with the following primary antibodies: Anti-SOX2 (1:1,000; cat. no. 3579), anti-BCL11A (1:1,000; cat. no. 75432), anti-Slug (1:1,000; cat. no. 9585), anti-N-cadherin (1:1,000; cat. no. 13116), anti-vimentin (1:1,000; cat. no. 5741), anti-E-cadherin (1:1,000; cat. no. 14472), anti-SKIL (1:1,000; cat. no. 4973), anti-TAZ (1:1,000; cat. no. 83669), anti-CTGF (1:1,000; cat. no. 86641) and anti-GAPDH (1:10,000; cat. no. 5174) at $4^{\circ} \mathrm{C}$ overnight. All primary antibodies were from Cell Signaling Technology, Inc. Then, the membranes were washed with TBST buffer and incubated with goat anti-rabbit $\operatorname{IgG}$ antibody tagged with horseradish peroxidase (1:10,000; cat. no. 4414; Cell Signaling Technology, Inc.) at room temperature for $2 \mathrm{~h}$. The membranes were scanned using an Odyssey Imaging system and analysed with the Odyssey v2.0 software (LI-COR Biosciences).

Luciferase reporter assay. The binding elements of miRNA-574-5p in BCL11A and SOX2 were predicted by Starbase (http://starbase.sysu.edu.cn). The wild-type (WT) BCL11A, mutant (Mut) BCL11A, WT SOX2 and Mut SOX2 were synthesized and inserted into the pGL3 vector (YouBio) to construct pGL3-BCL11A-WT, pGL3-BCL11A-Mut, pGL3-SOX2-WT and pGL3-SOX2-Mut, respectively, which were obtained from Shanghai GenePharma Co., Ltd. Then, the recombinant plasmids were transfected into cells with NC mimic or miR-574-5p mimics using Lipofectamine 3000 (Invitrogen; Thermo Fisher Scientific, Inc.). A Dual-Luciferase ${ }^{\mathrm{TM}}$ Reporter (DLR ${ }^{\mathrm{TM}}$ ) assay system (Promega 
Table I. shRNA target and mimics sequences.

shRNA or mimic

Target sequence $\left(5^{\prime}-3^{\prime}\right)$

shNC

BCL11A shRNA\#1

BCL11A ShRNA\#2

BCL11A shRNA\#3

miR-574 mimic sense

miR-574 mimic antisense

Mimic NC sense

Mimic NC antisense

GTTCTCCGAACGTGTCACGT

GCCTCTTGAAGCCATTCTTAC

GGAACACATAGCAGATAAACT

GGATTTCTCTAGGAGACTTAG

UGAGUGUGUGUGUGUGAGUGUGU

ACACUCACACACACACACUCAUU

UUCUCCGAACGUGUCACGUTT

ACGUGACACGUUCGGAGAATT

sh, short hairpin; NC, negative control; miR-574, microRNA-574; BCL11A, B-cell lymphoma/leukaemia $11 \mathrm{~A}$.

Table II. Primers used for reverse transcription-quantitative PCR.

\begin{tabular}{lll}
\hline Gene & Forward primer $\left(5^{\prime}-3^{\prime}\right)$ & Reverse primer $\left(5^{\prime}-3^{\prime}\right)$ \\
\hline miR-574-5p & GCCTGAGTGTGTGTGTGTGA & GTGCAGGGTCCGAGGT \\
BCL11A & ATGCGAGCTGTGCAACTATG & CAACACTCGATCACTGTGCC \\
SOX2 & CATGTCCCAGCACTACCAGA & TTTGAGCGTACCGGGTTTTC \\
U6 & CTCGCTTCGGCAGCACA & AACGCTTCACGAATTTGCGT \\
GAPDH & ACAAGATGGGCTTCATCCAC & CTCCATGCTGTCCTGTCTGA
\end{tabular}

miR-574-5p, microRNA-574-5p; SOX2, SRY (sex determining region Y)-box 2; BCL11A, B-cell lymphoma/leukaemia 11A.

Corporation) was applied for luciferase activity analysis. The activity of normalized reporter was expressed as the firefly luciferase value divided by the renilla luciferase value, and normalized to the control vector activity.

Animal experiments. This research was approved by the Ethics Committee of Xiangya Hospital affiliated to Central South University (approval no. 201803245). In total, 20 male BALB/c nude mice (4-6 weeks old) were obtained from SLAC Laboratory Animal Co. Ltd, (Hunan, China). Mice were raised in pathogen-free conditions at $20-25^{\circ} \mathrm{C}, 40-70 \%$ humidity, 15 times/h ventilation and a $12 \mathrm{~h}$ light/ $12 \mathrm{~h}$ dark cycle, with access to food and water ad libitum. For the xenograft assay, the mice were transplanted subcutaneously with MDA-MB-231 cells (transfected with NC mimic or miR-574-5p mimics) into the right flank and then housed until the tumour volume reached $\sim 200 \mathrm{~mm}^{3}$. Tumour size was measured every 5 days using the following formula: Length $\mathrm{x}$ width ${ }^{2} \mathrm{x} 0.5$. On the 30 th day, the mice were sacrificed and the tumour tissue was photographed. For the in vivo metastasis assay, $5 \times 10^{5}$ MDA-MB-231 cells (transfected with NC mimic or miR-574-5p mimics) were injected into the tail veins of nine mice. On the 30th day, the mice were sacrificed and the lung tissues were photographed. The number of pulmonary nodules was counted.

Haematoxylin-eosin staining was used for pulmonary migration analysis. Tissues were fixed by $4 \%$ formalin at $25^{\circ} \mathrm{C}$ for $24 \mathrm{~h}$, embedded in paraffin and cut into $5-\mu \mathrm{m}$ slices. The slices were stained with hematoxylin (Beyotime Institute of Biotechnology) for $5 \mathrm{~min}$ at $25^{\circ} \mathrm{C}$ and eosin (Beyotime
Institute of Biotechnology) for $3 \mathrm{~min}$ at $25^{\circ} \mathrm{C}$. The slices were then blocked with neutral gum at $25^{\circ} \mathrm{C}$ for $24 \mathrm{~h}$ and imaged using a light microscope (magnification, x100).

Statistical analysis. Statistical analysis was performed using GraphPad Prism 6.0 software (GraphPad Software, Inc.). Three independent experiments were performed, and data are expressed as the mean \pm standard deviation. Student's t-test was performed for comparisons between two groups, while one-way ANOVA followed by Tukey's post-hoc test was performed for multiple groups. $\mathrm{P}<0.05$ was considered to indicate a statistically significant difference.

\section{Results}

Expression of miR-574-5p in BC tissues and cells. The RT-qPCR results revealed that the expression level of miR-574-5p was significantly lower in tumour tissue compared with in the adjacent tissue of 45 patients (Fig. 1A). Subsequently, miR-574-5p expression levels in TNBC $(n=29)$ and non-TNBC $(n=16)$ were compared. As presented in Fig. 1B, miR-574-5p expression level in TNBC was significantly lower compared with that in non-TNBC. Furthermore, the expression of miR-574-5p was lower in both TNBC and non-TNBC cell lines (MDA-MB-436, MDA-MB-231, MDA-MB-468) compared with that in the normal breast epithelial cell line MCF10A (Fig. 1C). In summary, the expression of miR-574-5p was shown to be lower in both $\mathrm{BC}$ tissues and cells, particularly in TNBC tissue. 
A

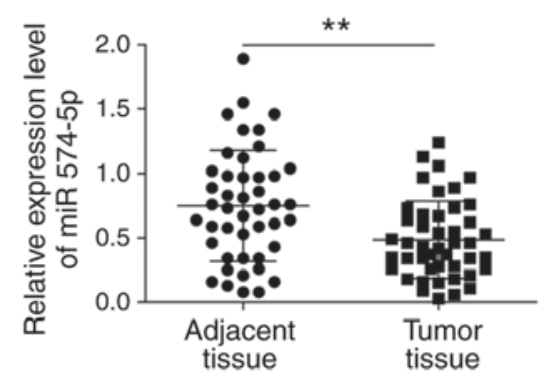

B

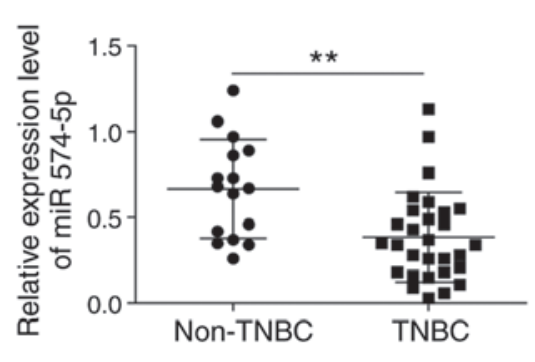

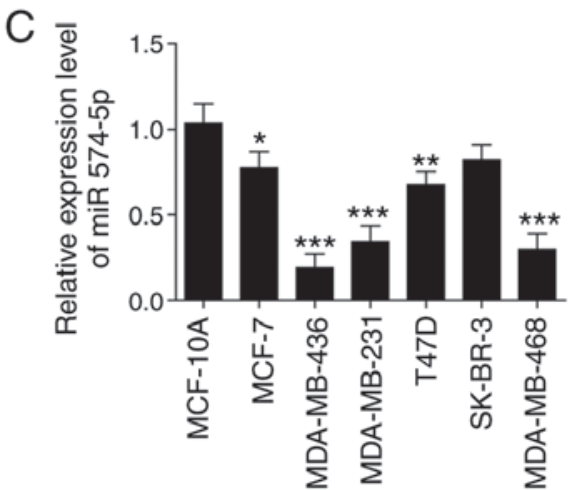

Figure 1. Expression of miR-574-5p in BC tissues and cells. Reverse transcription-quantitative PCR was performed to analyse the differences in miR-574-5p expression between (A) tumour tissue and adjacent tissue, (B) TNBC tissue and non-TNBC tissue, and (C) normal breast epithelial cells and BC cells. Data are presented as the mean \pm standard deviation from at least three independent experiments. ${ }^{*} \mathrm{P}<0.05,{ }^{* *} \mathrm{P}<0.01,{ }^{* * * *} \mathrm{P}<0.001$. miR-574-5p, microRNA-574-5p; $\mathrm{BC}$, breast cancer; TNBC, triple-negative breast cancer.

miR-574-5p inhibits proliferation, migration and EMT in TNBC cells. To explore the effects of miR-574-5p on TNBC progression, miR-574-5p was overexpressed in TNBC cells via transfection with miR-574-5p mimics or NC mimic. Compared with the NC mimic group, miR-574-5p expression was significantly increased in the miR-575-5p mimic-transfected group (Fig. 2A). The MTT assay revealed that cell viability was significantly suppressed in cells that overexpressed miR-574-5p (Fig. 2B and C). A colony formation assay was performed to detect the effect of miR-574-5p overexpression on colony formation. The results demonstrated that the colony formation ability of miR-574-5p-overexpressing cells was significantly inhibited compared with that of cells transfected with NC mimic (Fig. 2D). In addition, Transwell assays showed that miR-574-5p overexpression significantly inhibited cell migration and invasion (Fig. 2E and F). The effects of miR-574-5p on the expression of EMT-related proteins, E-cadherin, vimentin, Slug and N-cadherin, were analysed by western blotting. Overexpression of miR-574-5p significantly inhibited the expression of Slug, N-cadherin and vimentin, and significantly increased the expression of E-cadherin (Fig. 2G). Taken together, these findings suggested that miR-574-5p inhibits proliferation, migration and EMT in TNBC cells.

miR-574-5p targets BCL11A and SOX2 to inhibit the SKIL/TAZ/CTGF axis. To explore the mechanism by which miR-574 regulates the malignant phenotype, the present study examined the proteins involved in the SKIL pathway. The results of the western blot analysis revealed that overexpression of miR-574-5p significantly decreased the expression of SKIL, TAZ and CTGF (Fig. 3A). Next, how miR-574 regulates SKIL was examined. The effect of miR-574-5p overexpression on BCL11A and SOX2 expression levels was investigated, which have been reported to regulate SKIL transcription (22). The data demonstrated that the expression levels of BCL11A and SOX2 in cells transfected with miR-574-5p mimics were significantly lower compared that in control cells (Fig. 3B). Additionally, the relative mRNA expression levels of BCL11A and SOX2 in cells transfected with miR-574-5p mimics were significantly lower compared with in the NC mimic group (Fig. 3C and D). Bioinformatics prediction revealed that miR-574-5p targets the 3'-untranslated regions (3'-UTRs) of BCL11A and SOX2 (Fig. 3E). Subsequently, a luciferase assay was used to verify that miR-574-5p binds directly to the 3'-UTRs of BCL11A and SOX2. In the WT group, miR-574-5p overexpression significantly inhibited the luciferase activity of the reporter gene containing the WT BCL11A and SOX2 3'-UTR fragments, whereas for the Mut group, there was no significantly difference in luciferase activity between the NC mimic and miR-574-5p mimic groups, demonstrating that miR-574-5p binds directly to the predicted sequences of BCL11A and SOX2 (Fig. 3F and G). These combined data suggested that miR-574-5p targets BCL11A and SOX2, which may lead to miR-574-5p-mediated inhibition of the SKIL/TAZ/CTGF axis.

miR-574-5p inhibits tumorigenesis and lung metastasis in vivo. As presented in Fig. 4A-C, miR-574-5p overexpression significantly inhibited tumour size and weight in vivo. Additionally, miR-574-5p overexpression inhibited pulmonary migration and significantly reduced the number of metastatic nodules (Fig. 4D-F). Hence, it can been suggested that miR-574-5p may inhibit tumorigenesis and lung metastasis in vivo.

Inhibition of proliferation, migration and EMT by miR-574-5p in TNBC cells is at least partly dependent on targeting SOX2 and BCL11A. Co-overexpression of miR-574-5p and SOX 2 or BCL11A was performed by co-transfection with miR-574-5p mimics and pcDNA3.1-SOX2 or pcDNA3.1-BCL11A in MDA-MB-231 and MDA-MB-468 cells. Transfection efficiency is presented in Fig. S1A and B. Western blot analysis revealed that co-overexpression of miR-574-5p and SOX2 or BCL11A significantly reversed the inhibition of SKIL by miR-574-5p (Fig. 5A and B). Overexpression of SOX2 restored both BCL11A and SOX2 expression (Fig. 5A and B). However, BCL11A overexpression only restored its own expression and had no effect on the expression of SOX2 (Fig. 5A and B). Co-overexpression of miR-574-5p and SOX2 or BCL11A restored the inhibition of cell viability by miR-574-5p (Fig. 5C and D). miR-574-5p overexpression inhibited cell migration ability, while overexpression of miR-574-5p and SOX2 or BCL11A restored migration (Fig. 5E). Furthermore, western blot analysis demonstrated that co-overexpression of 
A

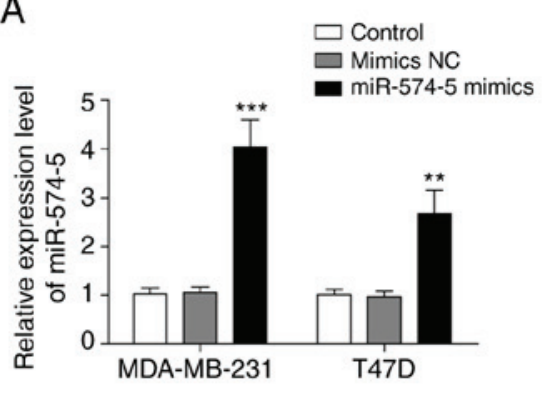

D

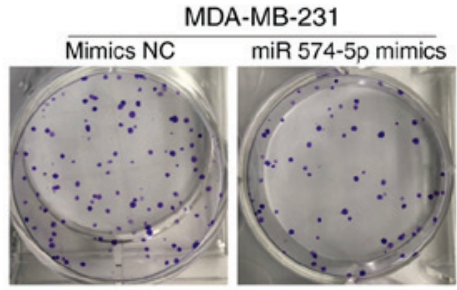

B
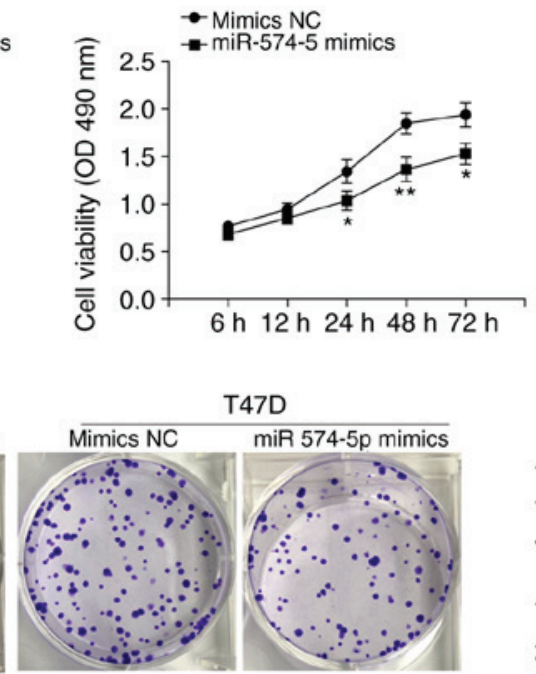

C

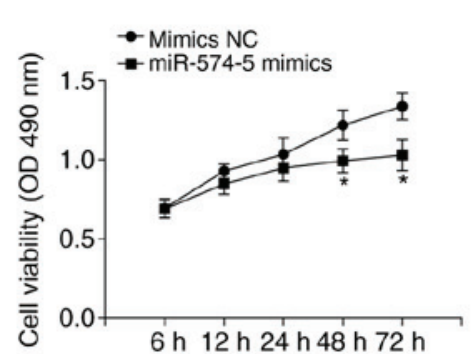

E
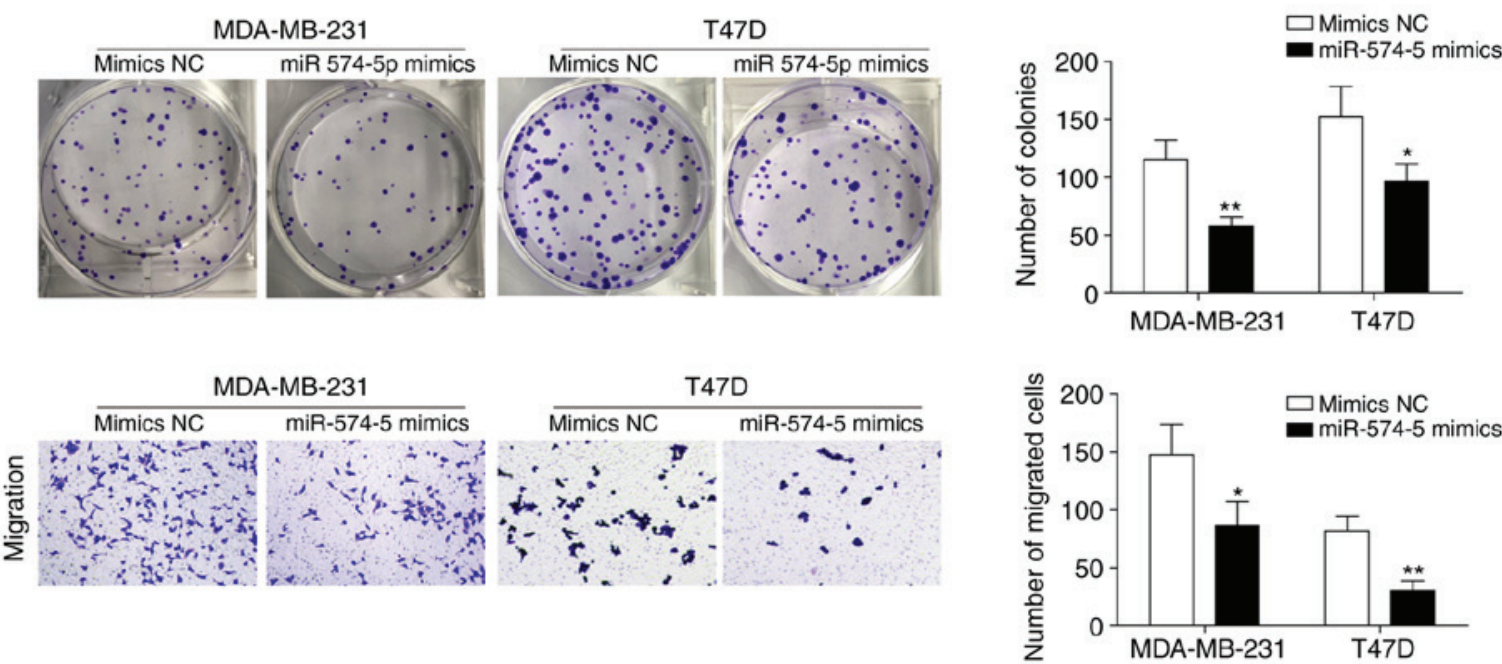

$\mathrm{F}$
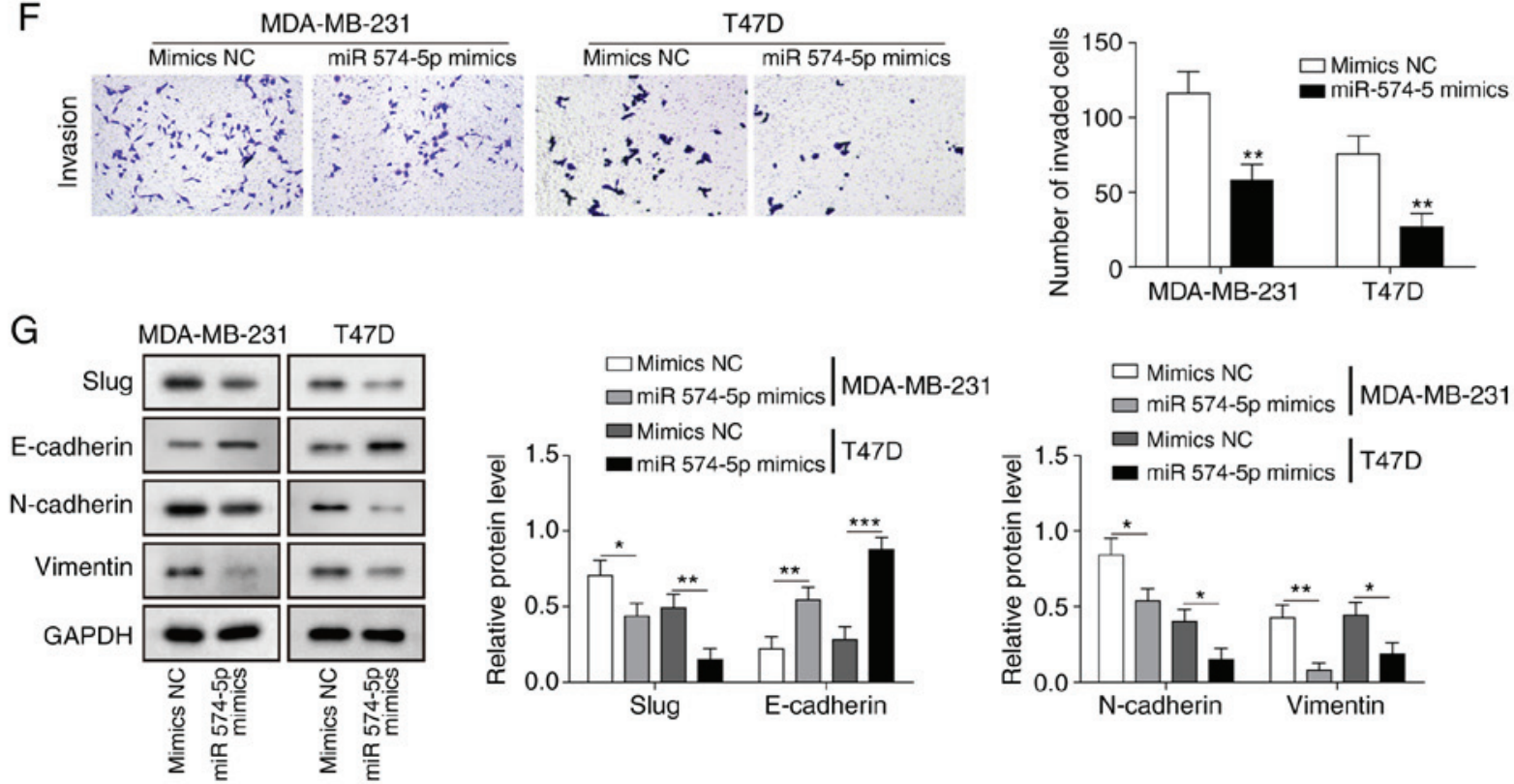

Figure 2. miR-574-5p inhibits the proliferation, migration and EMT of TNBC cells. (A) Reverse transcription-quantitative PCR was used to assess the relative miR-574-5p expression in MDA-MB-231 and MDA-MB-468 cells transfected with miR-574-5p mimics or NC mimic. MTT assay was performed to measure cell viability in (B) MDA-MB-231 and (C) MDA-MB-468 cells transfected with miR-574-5p mimics or NC mimic at 6, 12, 24, 48 and 72 h. (D) Cell colony formation ability in MDA-MB-231 and MDA-MB-468 cells transfected with miR-574-5p mimics or NC mimic was analysed by a colony formation assay. (E) Cell migration and (F) invasion ability in MDA-MB-231 and MDA-MB-468 cells transfected with miR-574-5p mimics or NC mimic was detected by Transwell experiments. Magnification, x100. (G) The effects of miR-574-5p on EMT-related proteins, E-cadherin, vimentin, Slug and N-cadherin, in MDA-MB-231 and MDA-MB-468 cells transfected with miR-574-5p mimics or NC mimic were analysed by western blotting. Data are presented as the mean \pm standard deviation from at least three independent experiments. ${ }^{*} \mathrm{P}<0.05,{ }^{* *} \mathrm{P}<0.01,{ }^{* * *} \mathrm{P}<0.001$ vs. mimics NC. miR-574-5p, microRNA-574-5p; TNBC, triple-negative breast cancer; NC, negative control; EMT, epithelial-mesenchymal transition; OD, optical density.

miR-574-5p and SOX2 or BCL11A reversed the inhibition of vimentin, Slug, and $\mathrm{N}$-cadherin expression and the promotion of E-cadherin expression by miR-574-5p (Fig. 5F and G). In summary, the inhibition of proliferation, migration and EMT by miR-574-5p in TNBC cells was at least partly dependent on targeting SOX2 and BCL11A. 

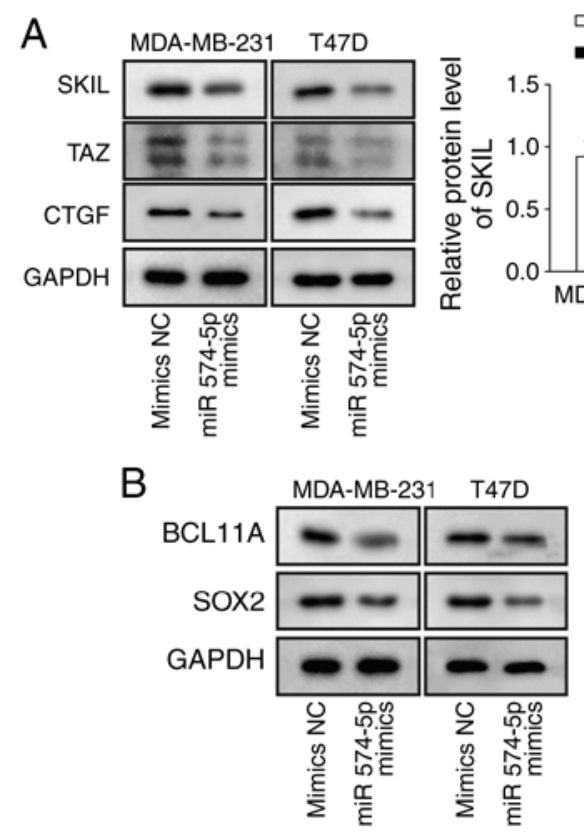

C

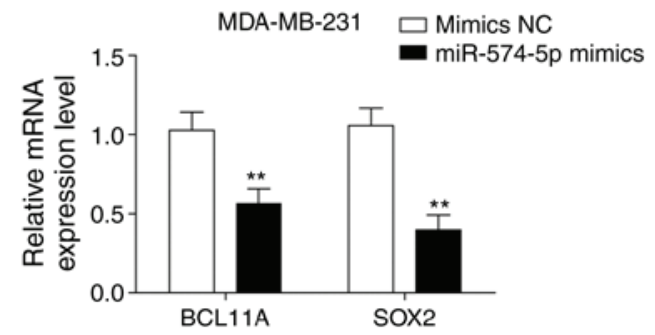

E

BCL11A 3'UTR WT 5' AAAUAUAGgaAaAUGCACACUCA 3'
hsa-miR-574-5p 3' UGUGUGaGUGUGUGUGUGUGAGU 5' BCL11A 3'UTR MUT 5' AAAUAUAGgAAAAUGGUGUGAGA 3'

$\mathrm{F}$

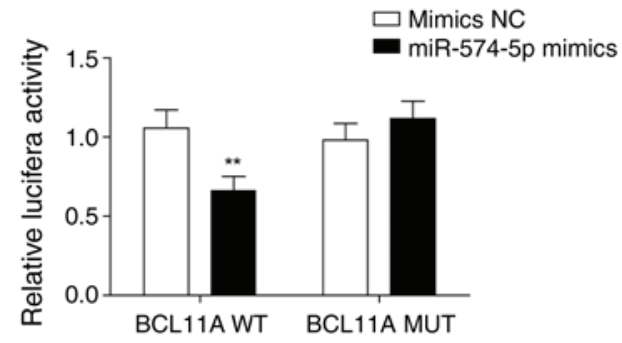

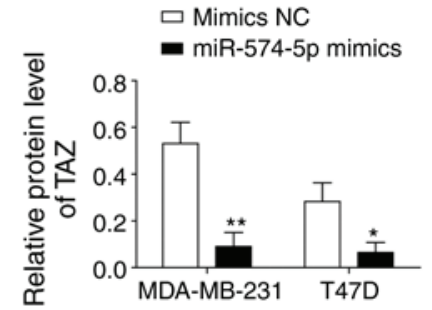

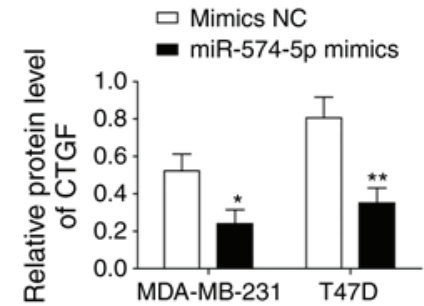

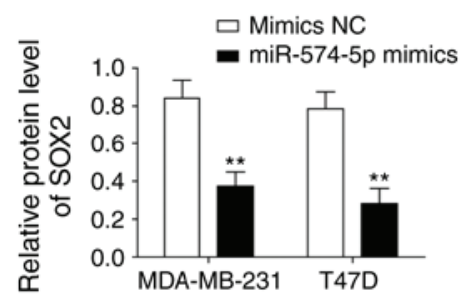

D

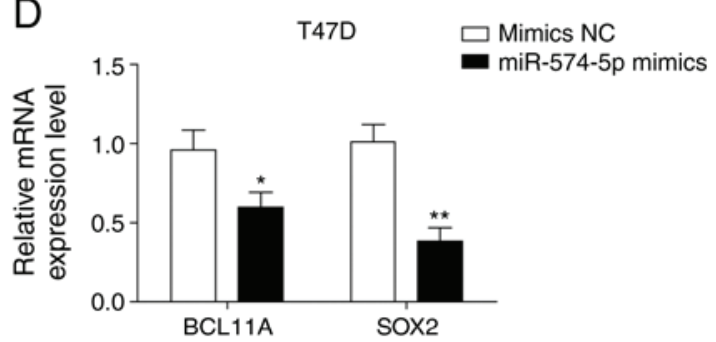

SOX2 3'UTR WT 5' GAACACCAAUCCCAUCCACACUCA 3' [11111

hsa-miR-574-5p 3' UGUGUGAGUGUGUGUGUGUGAGU 5'

SOX2 3'UTR MUT 5'GAACACCAAUCCCAUCGUGUGACA 3'

G

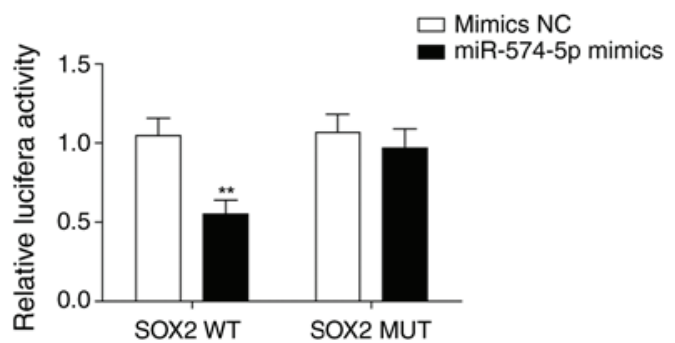

Figure 3. miR-574-5p targets BCL11A and SOX2 to inhibit the SKIL/TAZ/CTGF axis. Western blotting was used to assess the levels of (A) SKIL, TAZ, CTGF, (B) BCL11A and SOX2 in MDA-MB-231 and MDA-MB-468 cells transfected with miR-574-5p mimics or NC mimic. Reverse transcription-quantitative PCR was applied to evaluate the relative mRNA expression of BCL11A and SOX2 in (C) MDA-MB-231 and (D) MDA-MB-468 cells transfected with miR-574-5p mimics or NC mimic. (E) Binding sites of WT BCL11A and SOX2 3'-UTR to miR-574-5p and the mutation sites of BCL11A and SOX2. A luciferase assay was used to demonstrate that miR-574-5p directly targets the 3'-UTRs of (F) BCL11A and (G) SOX2. The luciferase activity of NC mimic-transfected cells was set to 1 . Data are presented as the mean \pm standard deviation from at least three independent experiments. ${ }^{*} \mathrm{P}<0.05,{ }^{* *} \mathrm{P}<0.01 \mathrm{vs}$. mimics NC. miR-574-5p, microRNA-574-5p; SOX2, SRY (sex determining region Y)-box 2; BCL11A, B-cell lymphoma/leukaemia 11A; SKIL, SKI like proto-oncogene; TAZ, transcriptional co-activator with PDZ-binding motif; CTGF, connective tissue growth factor; NC, negative control; WT, wild-type; 3'-UTR, 3'-untranslated region; MUT, mutated.

SOX2-mediated regulation of downstream oncogenes depends on BCL11A. As presented in Figs. 6A and S1C and D, the knockdown efficiency of BCL11A shRNAs was examined and shRNA\#3 was selected for subsequent experiments as it demonstrated the best knockdown efficiency in MDA-MB-231 cells. To further investigate the underlying interactive regulation of SOX2 and BCL11A, pcDNA3.1-SOX2 and shBCL11A were co-transfected into MDA-MB-231 and MDA-MB-468 cells. Western blot assays indicated that transfection with pcDNA3.1-SOX2 significantly upregulated the expression of SOX2, SKIL and BCL11A. BCL11A knockdown reversed this effect but had no effect on SOX2 expression (Fig. 6B and C). SOX2 overexpression significantly promoted cell viability and migration, while silencing BCL11A reversed this effect 
A
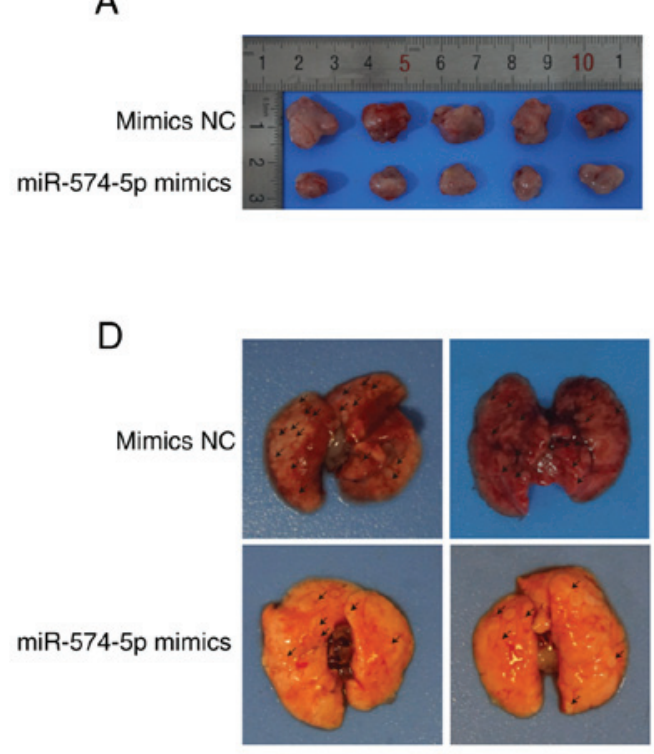

B

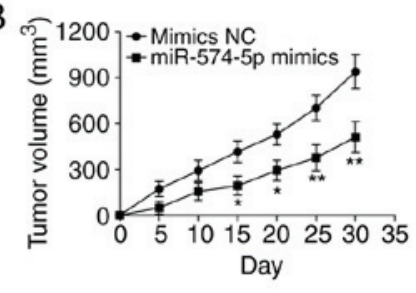

E

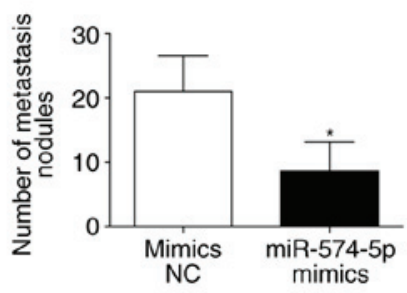

C

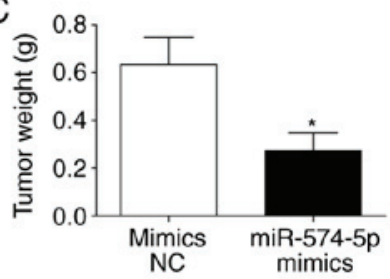

$\mathrm{F}$

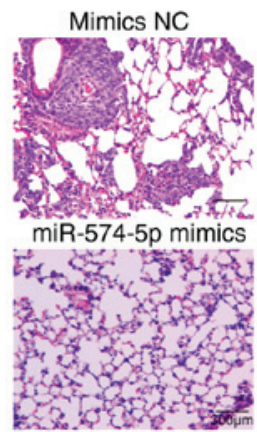

Figure 4. miR-574-5p inhibits tumorigenesis and lung metastasis in vivo. Ten nude mice were randomly divided into two groups. One group was injected with MDA-MB-231 cells transfected with NC mimic $(n=5)$ and the other group was injected with MDA-MB-231 cells transfected with miR-574-5p mimics ( $=5$ ). (A) Representative images of tumor in each group. Tumour sizes (B) and weights (C) were measured in the NC mimic group and the miR-574-5p mimics group. (D) Representative images of lung metastasis nodules from two mice in each group. The arrows indicate nodules. (E) The numbers of metastatic nodules were counted. (F) Haematoxylin and eosin staining was applied to analyse pulmonary migration. Magnification, $x 100$. Data are presented as the mean \pm standard deviation, $\mathrm{n}=5$. ${ }^{*} \mathrm{P}<0.05,{ }^{* *} \mathrm{P}<0.01$ vs. mimics NC. miR-574-5p, microRNA-574-5p; NC, negative control.

(Fig. 6D-F). Moreover, overexpression of SOX2 significantly increased the expression of Slug, vimentin and N-cadherin, and decreased the expression of E-cadherin, while BCL11A knockdown reversed this effect (Fig. 6G and H). Collectively, these results demonstrated that the oncogenic effect of SOX2 is partly dependent on BCL11A.

\section{Discussion}

TNBC is a subtype of breast cancer with poor prognosis that lacks specific markers for effective therapeutic targets (5). miRNAs are a class of short-chain non-coding RNAs that normally bind to the 3'-UTR of a target gene mRNA in a complementary manner to downregulate the expression of the target gene (33). Previous studies have confirmed the abnormal expression of multiple miRNAs in TNBC that are closely associated with the occurrence and development of TNBC $(34,35)$. Different miRNAs and their regulated target genes play different roles in the development and progression of TNBC, but the specific molecular mechanisms still require further study. The present study found that miR-574-5p was lower in TNBC tissues and cells. miR-574-5p inhibited the proliferation, migration and EMT of TNBC cells, and this was at least partly dependent on targeting SOX2 and BCL11A, which may involve the SKIL/TAZ/CTGF axis.

A number of studies have shown that miR-574-5p is involved in a variety of biological processes and has a wide range of effects (36-38). Wang et al (30) demonstrated that the long non-coding RNA linc-ZNF469-3 can promote breast cancer by inhibiting miR-574. However, to the best of our knowledge, the more detailed mechanism by which miR-574-5p regulates breast cancer progression remains unclear. The present study revealed a further mechanism of
miR-574-5p in TNBC, which was mediated by inhibition of the SOX2-induced SKIL/TAZ/CTGF axis. On the other hand, it was identified that miR-574-5p exerted a suppressive effect on TNBC by simultaneously targeting both SOX2 and BCL11A, suggesting that miRNAs can maximize their function by regulating multiple targets in the same pathway. Future work needs to focus on whether miR-574-5p targets other genes in this pathway.

BCL11A is a member of the B cell lymphoma/leukaemia family and is reported as an oncogene in TNBC $(39,40)$. Khaled et al (39) reported that high expression of BCL11A can promote the colony formation of mammary epithelial cells and stimulate the formation of tumours. The present study found that BCL11A mediated regulation of SOX2, which transcriptionally regulates the SKIL gene, thereby facilitating a malignant phenotype. SKIL is a cancer-promoting regulator mediated by SOX2 (9). SKIL can upregulate CTGF through the TAZ pathway, and CTGF is an important molecule that regulates cell migration and EMT (22). The present study identified that miR-574-5p inhibits the SKIL/TAZ/CTGF axis by inhibiting SOX2 and BCL11A and finally inhibits the proliferation, migration and EMT of TNBC. In addition, the present study also found that SOX2 overexpression could upregulate the expression of BCL11A, while the overexpression of BCL11A had no such effect on SOX2. This is consistent with the findings of Lazarus et al (9), which the present study has confirmed again in TNBC. SOX2 is an important oncogene for maintaining the embryonic development and multipotential differentiation of stem cells, and its expression level is related to the stem cell characteristics of various malignant cells $(11,41)$. The present study found that miR-574-5p targeted the expression of SOX2. Therefore, it can be hypothesized that miR-574-5p may also be able to regulate tumour cell stemness 
A

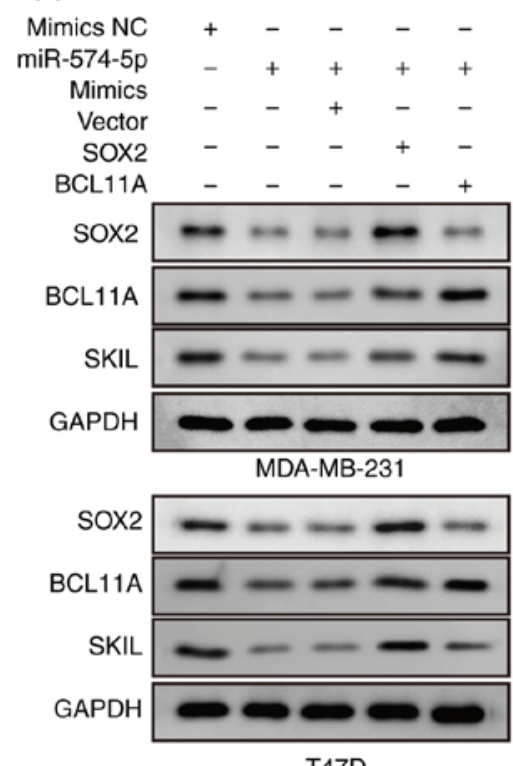

T47D
B

$\square$ Mimics NC

罚 miR-574-5p mimics

miR-574-5p mimics+vector

\& miR-574-5p mimics+SOX2

晨 miR-574-5p mimics+BCL11A
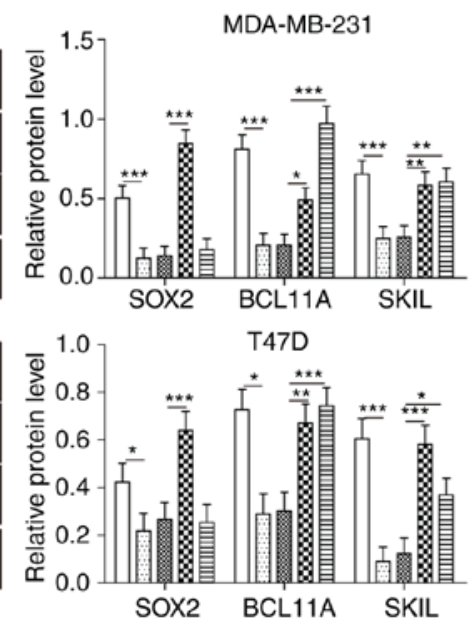

E

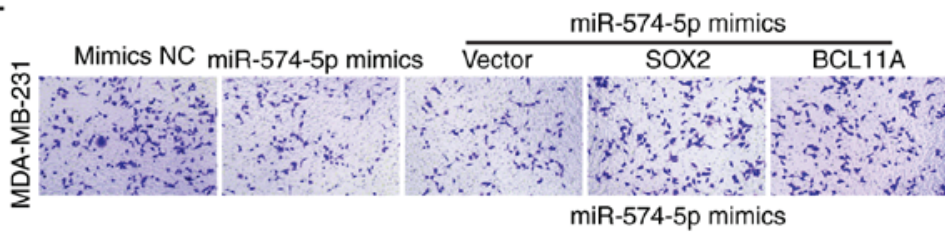

miR-574-5p mimics

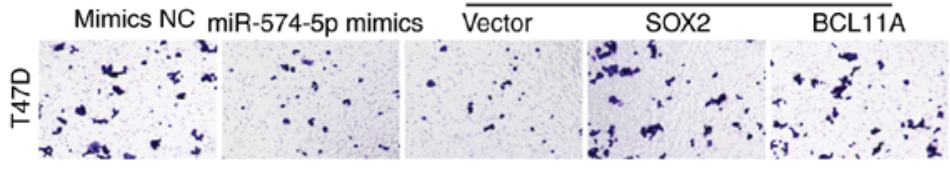

C

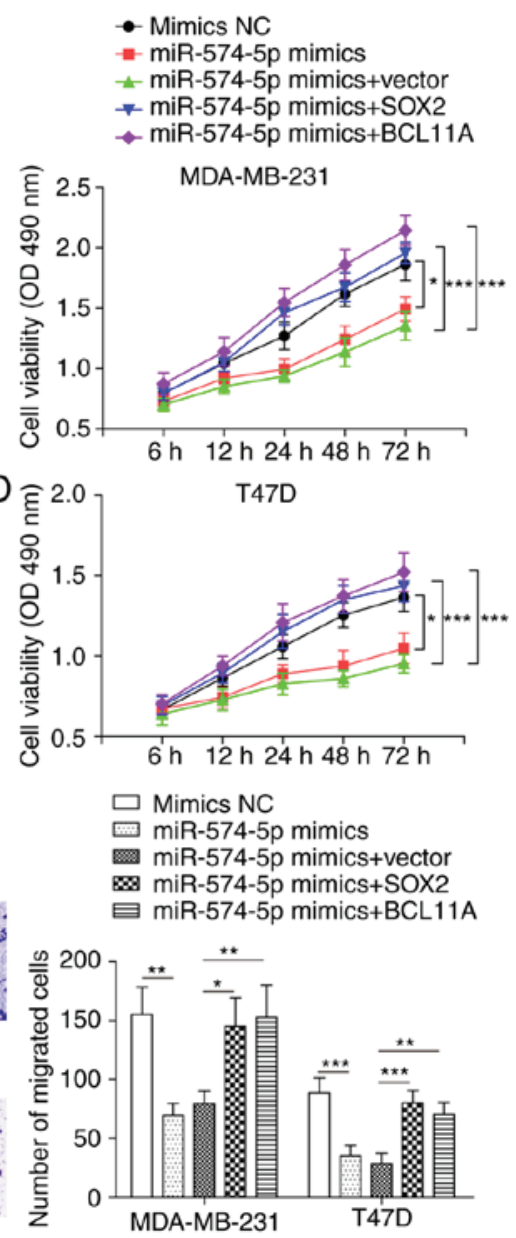

$\mathrm{F}$

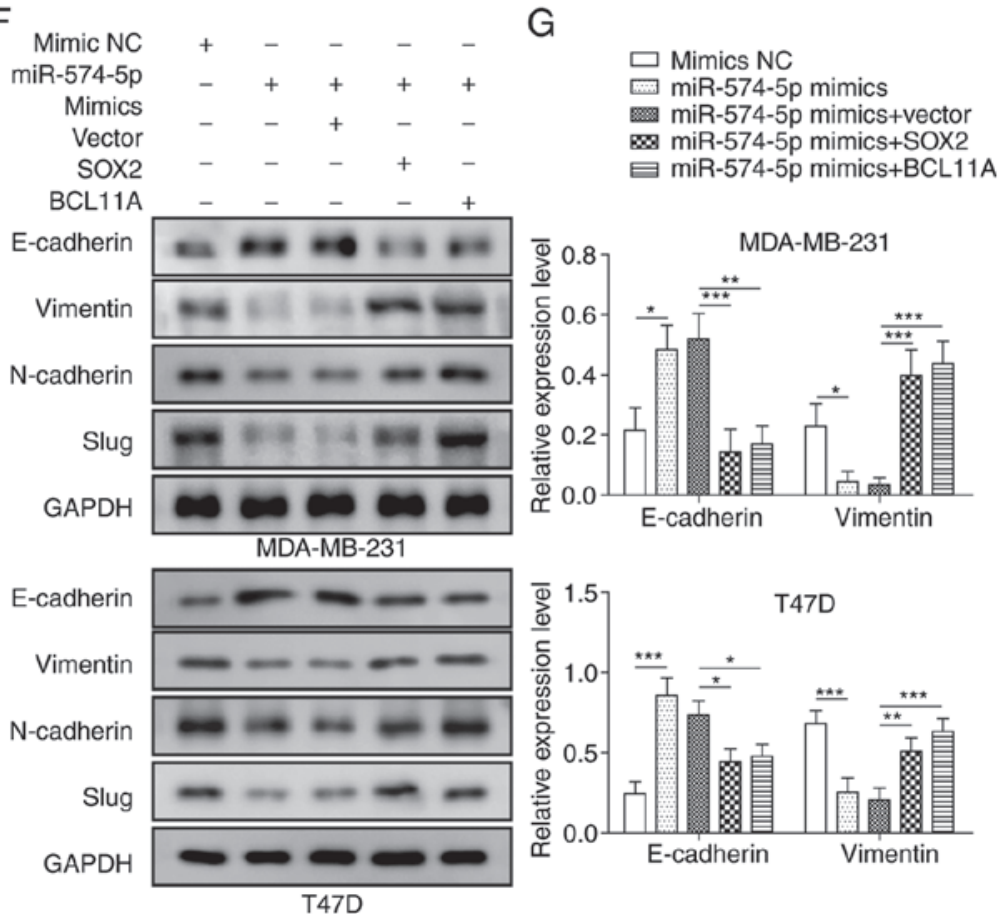

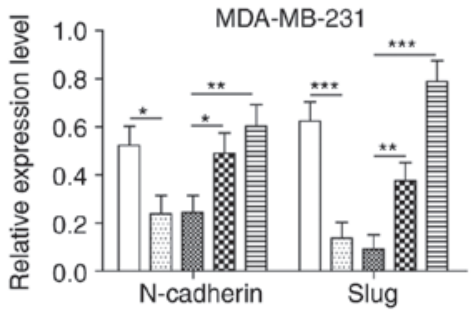

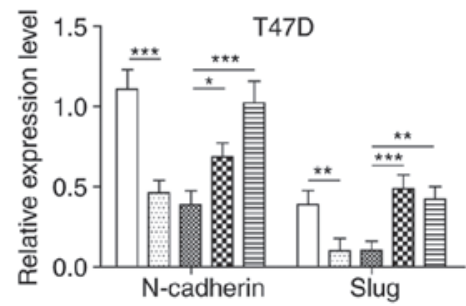

Figure 5. Inhibition of proliferation, migration and EMT by miR-574-5p in TNBC cells is dependent on SOX2 and BCL11A. MDA-MB-231 and MDA-MB-468 cells were divided into five groups: NC mimic, miR-574-5p mimic, miR-574-5p mimic + vector, miR-574-5p mimic + SOX2 and miR-574-5p mimic + BCL11A. (A and B) Western blotting was used to measure the expression of SOX2, BCL11A and SKIL. (C and D) The MTT assay was performed to assess cell viability at 6,12,24, 48 and $72 \mathrm{~h}$. (E) Transwell assay was used to analyse migration ability. Magnification, x100. (F and G) Western blotting was applied to measure the expression levels of E-cadherin, vimentin, Slug and N-cadherin. Data are presented as the mean \pm SD for three independent experiments. ${ }^{*} \mathrm{P}<0.05,{ }^{* * *} \mathrm{P}<0.01,{ }^{* * * *} \mathrm{P}<0.001$. miR-574-5p, microRNA-574-5p; EMT, epithelial-mesenchymal transition; OD, optical density; SOX2, SRY (sex determining region Y)-box 2; BCL11A, B-cell lymphoma/leukaemia 11A; SKIL, SKI like proto-oncogene; NC, negative control. 
A

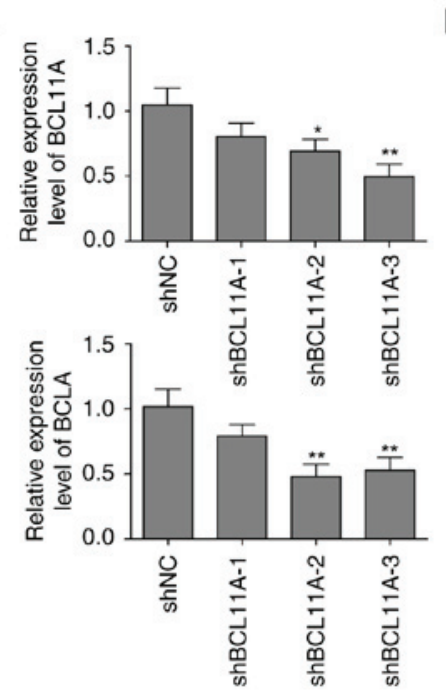

D

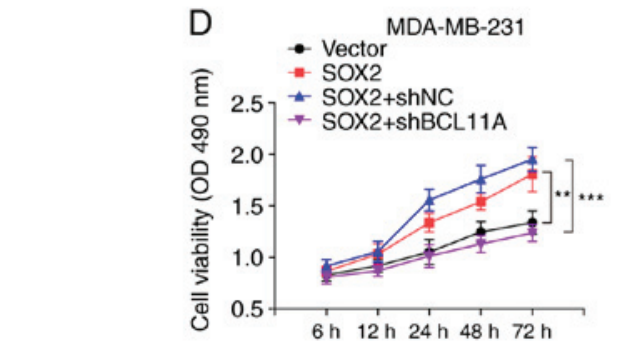

B Vector
SOX2
shNC

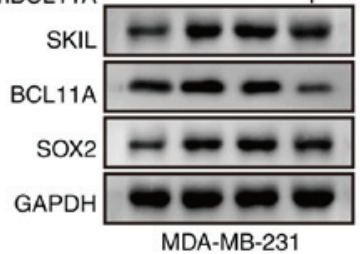

MDA-MB-231

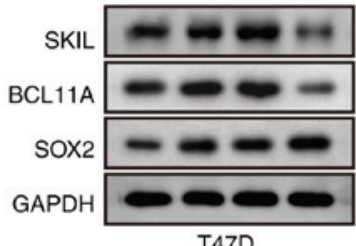

C MDA-MB-231

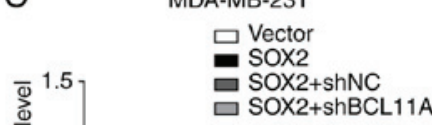

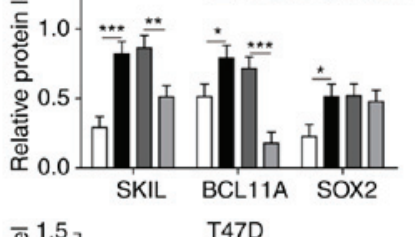

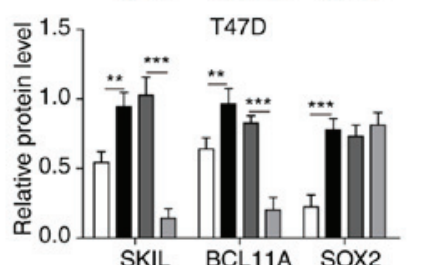

E
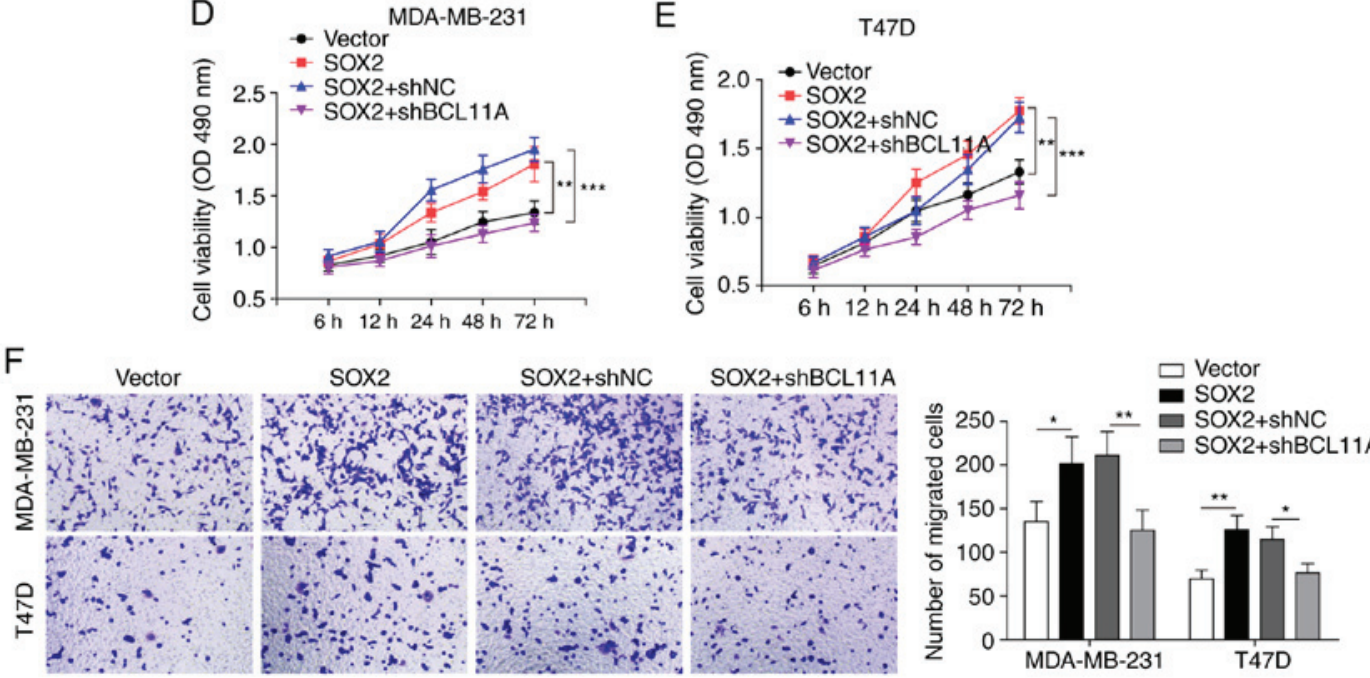

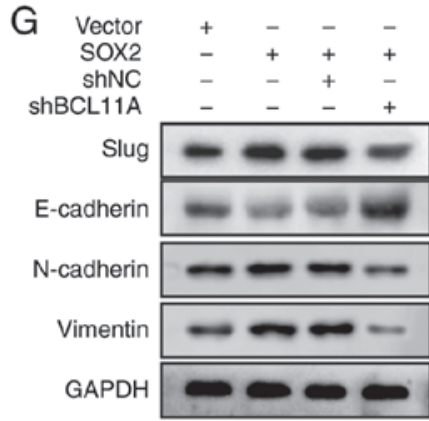

$\mathrm{H}$
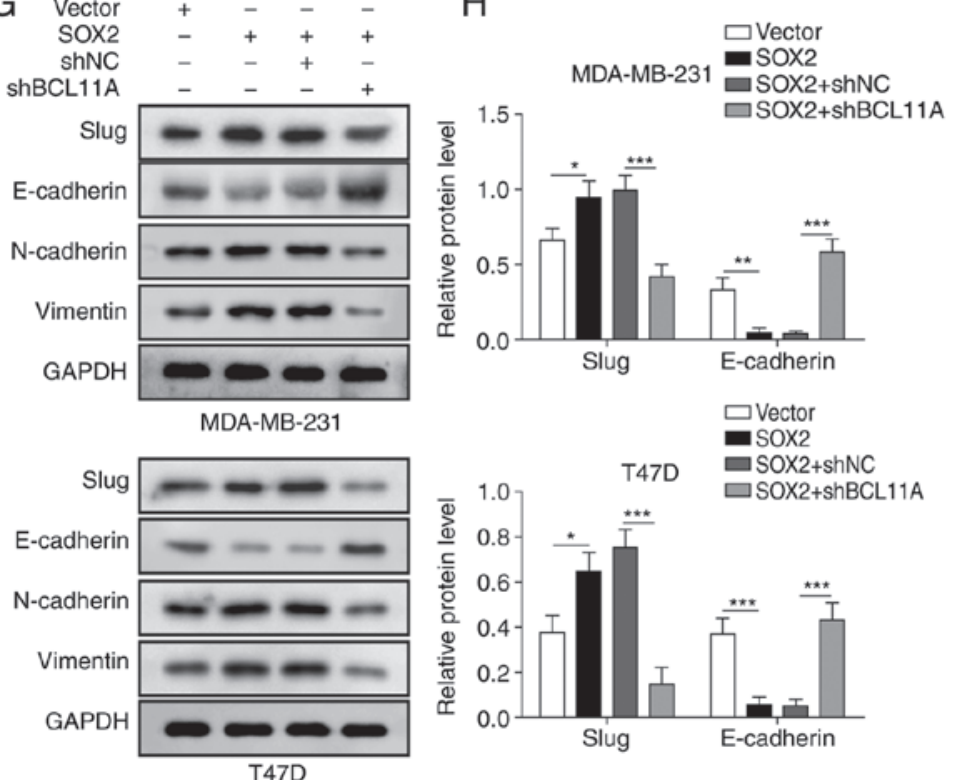

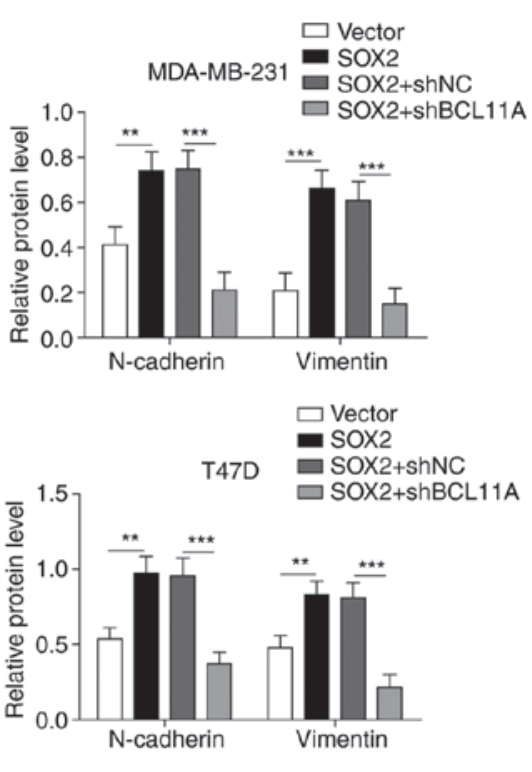

Figure 6. SOX2-mediated regulation of downstream oncogenes depends on BCL11A. MDA-MB-231 and MDA-MB-468 cells were divided into four groups: Vector, pcDNA3.1-SOX2, pcDNA3.1-SOX2 + shNC and pcDNA3.1-SOX2 + shBCL11A. (A) BCL11A-knockdown was performed with different shRNAs. (B and C) Western blotting was used to detect SOX2, SKIL and BCL11A levels. (D and E) The MTT assay was applied to analyse cell viability at 6, 12, 24, 48 and $72 \mathrm{~h}$. (F) Cell migration ability was analysed by Transwell assay. Magnification, x100. (G and H) Slug, vimentin, N-cadherin and E-cadherin levels were measured by western blotting. Data are presented as the mean \pm standard deviation for three independent experiments. ${ }^{*} \mathrm{P}<0.05,{ }^{* * *} \mathrm{P}<0.01,{ }^{* * * *} \mathrm{P}<0.001$. SOX2, SRY (sex determining region Y)-box 2; BCL11A, B-cell lymphoma/leukaemia 11A; SKIL, SKI like proto-oncogene; NC, negative control; sh, short hairpin; OD, optical density. 
by targeting SOX2; however, this requires further experimental validation. In addition, it was recognized that SOX2 may promote TNBC independent of SKIL; hence, a definite conclusion that the miR-574-5p/SOX2/BCL11A axis regulates the malignant phenotype of TNBC via SKIL/TAZ/CTGF cannot be made.

In conclusion, the present study identified the novel miR-574-5p/SOX2/BCL11A signalling axis in TNBC. It was demonstrated that miR $\square 574-5 p$ inhibited the proliferation, migration and EMT of TNBC, and this was at least partly dependent on directly targeting BCL11A and SOX2, which may therefore regulate the downstream SKIL/TAZ/CTGF axis. To the best of our knowledge, this is the first study to report the underlying mechanism between the miR-574-5p/BCL11A/SOX2 axis and the tumorigenesis of TNBC, which provides new insight into the progression of TNBC.

\section{Acknowledgements}

Not applicable.

\section{Funding}

This study was supported by the Science and Technology Project of Hunan Province (grant no. 2015JC3016) and the Scientific Research Project of Hunan Provincial Health and Family Planning Commission (grant no. B2016106).

\section{Availability of data and materials}

The datasets used and/or analysed during the current study are available from the corresponding author on reasonable request.

\section{Authors' contributions}

$\mathrm{KJZ}$ designed the study, performed the literature research, performed experimental studies, acquired the data, analysed the data, and prepared, edited and reviewed the manuscript. LG designed the study, performed the statistical analysis, reviewed the manuscript and is responsible for the integrity of the study. JQY performed the clinical and experimental studies. YH and XL performed the statistical analysis. NL edited the manuscript and performed the statistical analysis. FYC acquired the data of the clinical study and performed the statistical analysis of clinical study. All authors read and approved the manuscript.

\section{Ethics approval and consent to participate}

The present study was approved by the Ethics Committee of Xiangya Hospital Affiliated to Central South University (approval no. 201803377). Written informed consent was obtained from the participants. The animal study was approved by the Ethics Committee of Xiangya Hospital Affiliated to Central South University (approval no. 201803245).

\section{Patient consent for publication}

Not applicable.

\section{Competing interests}

The authors declare that they have no competing interests.

\section{References}

1. Torre LA, Bray F, Siegel RL, Ferlay J, Lortet-Tieulent J and Jemal A: Global cancer statistics, 2012. CA Cancer J Clin 65: 87-108, 2015

2. Wu Y,Zhang Z, Cenciarini ME, Proietti CJ, Amasino M, Hong T, Yang M, Liao Y, Chiang HC, Kaklamani VG, et al: Tamoxifen resistance in breast cancer is regulated by the EZH2-ER $\alpha$-GREB1 transcriptional axis. Cancer Res 78: 671-684, 2018.

3. Kumar P and Aggarwal R: An overview of triple-negative breast cancer. Arch Gynecol Obstet 293: 247-269, 2016.

4. Liu F, Zhuang L, Wu R and Li D: miR-365 inhibits cell invasion and migration of triple negative breast cancer through ADAM10. J BUON 24: 1905-1912, 2019.

5. Denkert C, Liedtke C, Tutt A and von Minckwitz G: Molecular alterations in triple-negative breast cancer-the road to new treatment strategies. Lancet 389: 2430-2442, 2017.

6. Yu Y, Wang J, Khaled W, Burke S, Li P, Chen X, Yang W, Jenkins NA, Copeland NG, Zhang S and Liu P: Bcl1la is essential for lymphoid development and negatively regulates p53. J Exp Med 209: 2467-2483, 2012.

7. Nakamura T, Yamazaki Y, Saiki Y, Moriyama M, Largaespada DA, Jenkins NA and Copeland NG: Evi9 encodes a novel zinc finger protein that physically interacts with BCL6, a known human B-cell proto-oncogene product. Mol Cell Biol 20: 3178-3186, 2000 .

8. Chen F, Luo N, Hu Y, Li X and Zhang K: MiR-137 suppresses triple-negative breast cancer stemness and tumorigenesis by perturbing BCL11A-DNMT1 interaction. Cell Physiol Biochem 47: 2147-2158, 2018.

9. Lazarus KA, Hadi F,Zambon E, Bach K, Santolla MF, Watson JK, Correia LL, Das M, Ugur R, Pensa S, et al: BCL11A interacts with SOX2 to control the expression of epigenetic regulators in lung squamous carcinoma. Nat Commun 9: 3327, 2018.

10. Boumahdi S, Driessens G, Lapouge G, Rorive S, Nassar D, Le Mercier M, Delatte B, Caauwe A, Lenglez S, Nkusi E, et al: SOX2 controls tumour initiation and cancer stem-cell functions in squamous-cell carcinoma. Nature 511: 246-250, 2014.

11. Mamun MA, Mannoor K, Cao J, Qadri F and Song X: SOX2 in cancer stemness: Tumor malignancy and therapeutic potentials. J Mol Cell Biol: Dec 5, 2018 (Epub ahead of print).

12. Tong B, Zeng J, Wu Y and Xiong W: Enhanced SOX2 expression in retinoblastoma tissues and peripheral blood is associated with the clinicopathological characteristics of the disease. Oncol Lett 9: 1244-1248, 2015.

13. Nomura N, Sasamoto S, Ishii S, Date T, Matsui M and Ishizaki R: Isolation of human cDNA clones of ski and the ski-related gene, sno. Nucleic Acids Res 17: 5489-5500, 1989.

14. Jahchan NS and Luo K: SnoN in mammalian development, function and diseases. Curr Opin Pharmacol 10: 670-675, 2010.

15. Shinozuka E, Miyashita M, Mizuguchi Y, Akagi I, Kikuchi K, Makino H, Matsutani T, Hagiwara N, Nomura T, Uchida E and Takizawa T: SnoN/SKIL modulates proliferation through control of hsa-miR-720 transcription in esophageal cancer cells. Biochem Biophys Res Commun 430: 101-106, 2013.

16. Kodigepalli KM, Anur P, Spellman P, Sims PJ and Nanjundan M: Phospholipid Scramblase 1, an interferon-regulated gene located at $3 q 23$, is regulated by SnoN/SkiL in ovarian cancer cells. Mol Cancer 12: 32, 2013.

17. Javelaud D, van Kempen L, Alexaki VI, Le Scolan E, Luo K and Mauviel A: Efficient TGF- $\beta /$ SMAD signaling in human melanoma cells associated with high c-SKI/SnoN expression. Mol Cancer 10: 2, 2011.

18. Makino Y, Yoon JH, Bae E, Kato M, Miyazawa K, Ohira T, Ikeda N, Kuroda M and Mamura M: Repression of Smad3 by Stat 3 and c-Ski/SnoN induces gefitinib resistance in lung adenocarcinoma. Biochem Biophys Res Commun 484: 269-277, 2017.

19. Sengupta S, Jana S, Biswas S, Mandal PK and Bhattacharyya A: Cooperative involvement of NFAT and SnoN mediates transforming growth factor- $\beta$ (TGF- $\beta$ ) induced EMT in metastatic breast cancer (MDA-MB 231) cells. Clin Exp Metastasis 30: 1019-1031, 2013.

20. Zhu Q, Krakowski AR, Dunham EE, Wang L, Bandyopadhyay A, Berdeaux R, Martin GS, Sun L and Luo K: Dual role of SnoN in mammalian tumorigenesis. Mol Cell Biol 27: 324-339, 2007. 
21. Band AM and Laiho M: SnoN oncoprotein enhances estrogen receptor- $\alpha$ transcriptional activity. Cell Signal 24: 922-930, 2012.

22. Zhu Q, Le Scolan E, Jahchan N, Ji X, Xu A and Luo K: SnoN antagonizes the hippo kinase complex to promote TAZ signaling during breast carcinogenesis. Dev Cell 37: 399-412, 2016.

23. Janse van Rensburg HJ, Azad T, Ling M, Hao Y, Snetsinger B, Khanal P, Minassian LM, Graham CH, Rauh MJ and Yang X: The hippo pathway component TAZ promotes immune evasion in human cancer through PD-L1. Cancer Res 78: 1457-1470, 2018

24. Harvey KF, Zhang $X$ and Thomas DM: The Hippo pathway and human cancer. Nat Rev Cancer 13: 246-257, 2013.

25. Krol J, Loedige I and Filipowicz W: The widespread regulation of microRNA biogenesis, function and decay. Nat Rev Genet 11: 597-610, 2010

26. Tian Y, Fu X, Li Q, Wang Y, Fan D, Zhou Q, Kuang W and Shen L: MicroRNA181 serves an oncogenic role in breast cancer via the inhibition of SPRY4. Mol Med Rep 18: 5603-5613, 2018.

27. Krutzfeldt J: Strategies to use microRNAs as therapeutic targets. Best Pract Res Clin Endocrinol Metab 30: 551-561, 2016

28. Peng G, Liao Y and Shen C: miRNA-429 inhibits astrocytoma proliferation and invasion by targeting BMI1. Pathol Oncol Res 23: 369-376, 2017.

29. Deng Z, Wang Y, Fang X, Yan F, Pan H, Gu L, Xie C, Li Y, Hu Y, Cao Y and Tang Z: Research on miRNA-195 and target gene CDK6 in oral verrucous carcinoma. Cancer Gene Ther 24: 282-288, 2017

30. Wang PS, Chou CH, Lin CH, Yao YC, Cheng HC, Li HY, Chuang YC, Yang CN, Ger LP, Chen YC, et al: A novel long non-coding RNA linc-ZNF469-3 promotes lung metastasis through miR-574-5p-ZEB1 axis in triple negative breast cancer. Oncogene 37: 4662-4678, 2018.

31. Huang $X$ and Yin YM: Updates of chinese society of clinical oncology (CSCO) guideline for breast cancer in 2018. Zhonghua Yi Xue Za Zhi 98: 1213-1217, 2018 (In Chinese).
32. Livak KJ and Schmittgen TD: Analysis of relative gene expression data using real-time quantitative PCR and the 2(-Delta Delta C(T)) method. Methods 25: 402-408, 2001

33. Shah MY and Calin GA: MicroRNAs as therapeutic targets in human cancers. Wiley Interdiscip Rev RNA 5: 537-548, 2014.

34. D'Ippolito E and Iorio MV: MicroRNAs and triple negative breast cancer. Int J Mol Sci 14: 22202-22220, 2013.

35. Chang YY, Kuo WH, Hung JH, Lee CY, Lee YH, Chang YC, Lin WC, Shen CY, Huang CS, Hsieh FJ, et al: Deregulated microRNAs in triple-negative breast cancer revealed by deep sequencing. Mol Cancer 14: 36, 2015.

36. Wang $X$, Lu X, Geng Z, Yang $G$ and Shi Y: LncRNA PTCSC3/miR-574-5p governs cell proliferation and migration of papillary thyroid carcinoma via wnt $/ \beta$-catenin signaling. J Cell Biochem 118: 4745-4752, 2017.

37. Lai Z, Lin P, Weng X, Su J, Chen Y, He Y, Wu G, Wang J, Yu Y and Zhang L: MicroRNA-574-5p promotes cell growth of vascular smooth muscle cells in the progression of coronary artery disease. Biomed Pharmacother 97: 162-167, 2018.

38. Ku T, Li B, Gao R, Zhang Y, Yan W, Ji X, Li G and Sang N: NF- $\kappa$ B-regulated microRNA-574-5p underlies synaptic and cognitive impairment in response to atmospheric PM2.5 aspiration. Part Fibre Toxicol 14: 34, 2017.

39. Khaled WT, Choon Lee S, Stingl J, Chen X, Raza Ali H, Rueda OM, Hadi F, Wang J, Yu Y, Chin SF, et al: BCL11A is a triple-negative breast cancer gene with critical functions in stem and progenitor cells. Nat Commun 6: 5987, 2015.

40. Errico A: Genetics: BCL11A-targeting triple-negative breast cancer? Nat Rev Clin Oncol 12: 127, 2015.

41. Liu DW, Zhang JH, Liu FX, Wang XT, Pan SK, Jiang DK, Zhao ZH and Liu ZS: Silencing of long noncoding RNA PVT1 inhibits podocyte damage and apoptosis in diabetic nephropathy by upregulating FOXA1. Exp Mol Med 51: 88, 2019. 